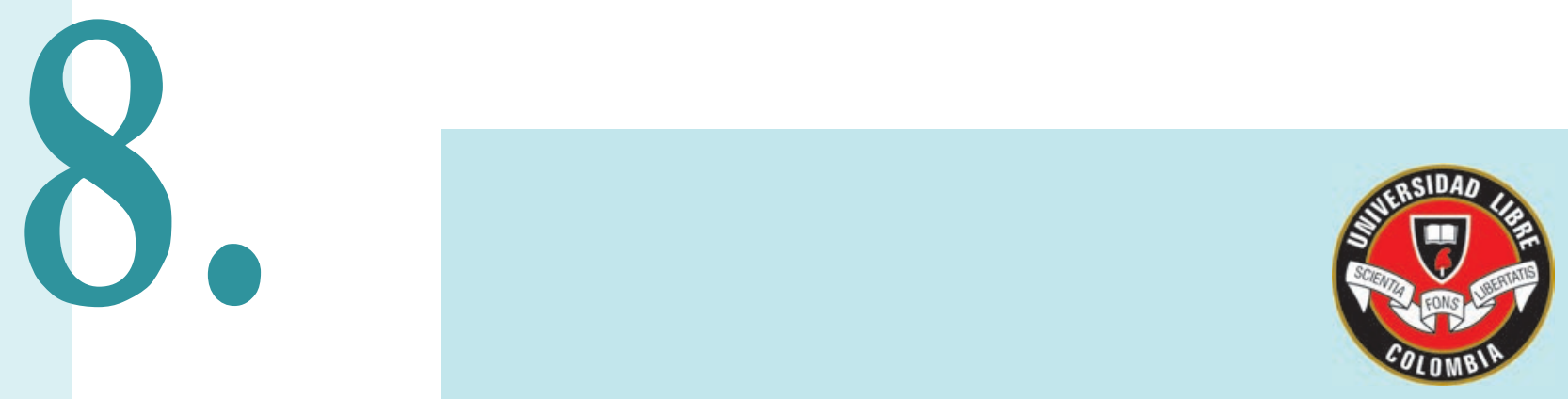

La Calidad Académica, un Compromiso Institucional

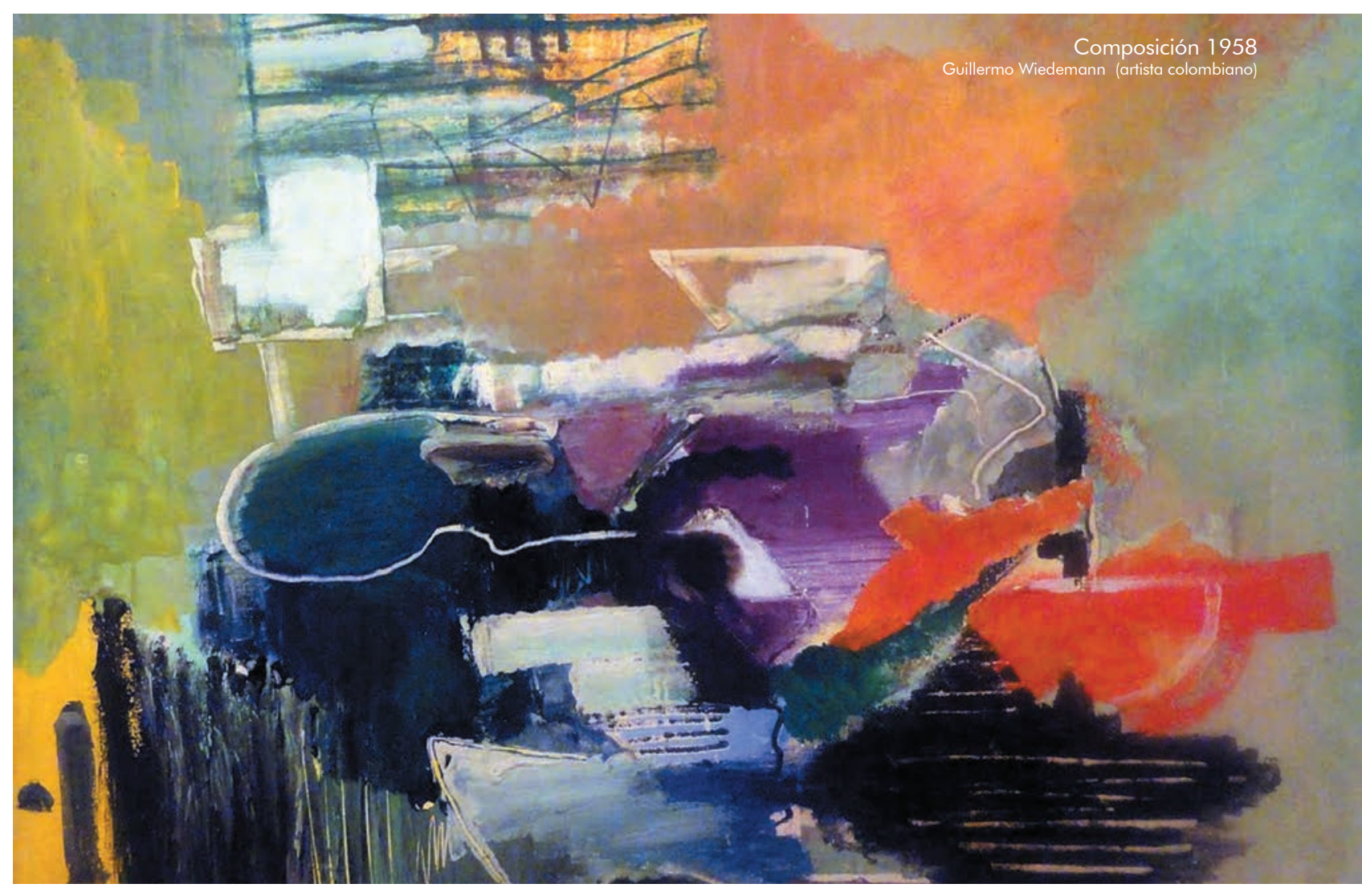

Barbosa R., David; Piñeros, Rafael y Noguera, Ángela (2013). Retos actuales del gobierno corporativo en torno a lo creación de valor. Criterio Libre, 11 (19) 187-204

ISSN 1900-0642

Retos actuales del gobiemo corporativo en torno a la creación de valor

David Barbosa Ramírez • Rafael Piñeros - Ángela Noguera 


\section{RETOS ACTUALES \\ DEL GOBIERNO CORPORATIVO EN TORNO A LA CREACIÓN DE VALOR*}

CURRENT CHALLENGES OF CORPORATE GOVERNMENT AROUND THE CREATION OF VALUE DESAFIOS ATUAIS DE GOVERNO CORPORATIVO EM TORNO DA CRIAÇÃO DE VALOR

LES DÉFIS ACTUELS DU GOUVERNEMENT CORPORATIF DANS LA CRÉATION DE VALEURS

\section{DAVID BARBOSA RAMÍREZ RAFAEL PIÑEROS§ ÁNGELA NOGUERA}

\footnotetext{
Artículo de reflexión, resultado de impartir los cursos Introducción a la Administración, Liderazgo, Pensamiento Estratégico y Gobierno de Empresa en programas de pregrado y posgrado.

Reflection article, result of imparting Administration, Leadership, Strategic Thinking and Corporate Government introductory courses to pre-graduate and post-graduate programs. Artigo de reflexão, resultado da atividade de docência em cursos de introdução à Administração, Liderança, Pensamento Estratégico e Governo de Empresa em programas de graduação e pós-graduação.

Article de réflexion, c'est le résultat d'avoir donné les cours d'Introduction à la gestion, le leadership, la réflexion stratégique et de gouvernement d'entreprise dans les programmes de premier cycle et des cycles supérieurs.

‡ Médico y cirujano, Pontificia Universidad Javeriana, Bogotá, Colombia. Abogado, Universidad del Rosario, Bogotá. Especialista en Derecho Laboral, Pontificia Universidad Javeriana, Bogotá. Magíster en Administración, Universidad de los Andes, Bogotá. Candidato a doctor en Ciencias de la Dirección, Universidad del Rosario, Bogotá. Profesor principal, Universidad del Rosario. Grupo de Investigación en Perdurabilidad Empresarial, GIPE. david.barbosa@urosario.edu.co MD and Surgeon, Pontificia Universidad Javeriana, Bogotá, Colombia. Attorney, Universidad del Rosario, Bogotá. Specialist in Labor Law, Pontificia Universidad Javeriana, Bogotá. Master in Administration, Universidad de los Andes, Bogotá. PhD candidate in Direction Science, Universidad del Rosario, Bogotá. Lead Professor, Universidad del Rosario. Research Group Member in Corporate Durability, GIPE. david.barbosa@urosario.edu.co

Médico e cirurgião, Pontificia Universidad Javeriana, Bogotá, Colômbia. Advogado, Universidad del Rosario, Bogotá. Especialista em Direito do Trabalho, Pontificia Universidad Javeriana, Bogotá. Mestre em Administração, Universidad de los Andes, Bogotá. Doutorando em Ciências da Direção, Universidad del Rosario, Bogotá. Professor titular, Universidad del Rosario. Grupo de pesquisa sobre Perdurabilidade empresarial, GIPE.david.barbosa@urosario.edu.co

Médecin et chirurgien - Pontificia Universidad Javeriana, Bogota, Colombie. Avocat, Universidad del Rosario, Bogotá. Spécialiste en droit du travail, Pontificia Universidad Javeriana, Bogota. Master of Management, Universidad de los Andes, Bogotá. Candidat au doctorat en sciences de gestion, Universidad del Rosario, Bogotá. Maître de conférences, Université de Rosario. Durabilité Business Research Group, GIPE. david.barbosa@ urosario.edu.co

$\S$ Magister en Dirección y Gerencia de Empresas, Universidad del Rosario, Colombia; Administrador de Empresas, Universidad del Rosario, Colombia; Profesor auxiliar, Universidad del Rosario; Grupo de Investigación en Perdurabilidad Empresarial, GIPE; rafael.pineros@urosario.edu.co
}

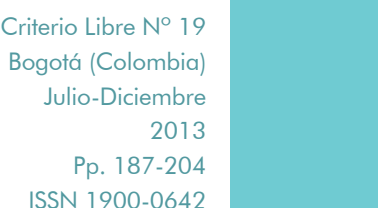




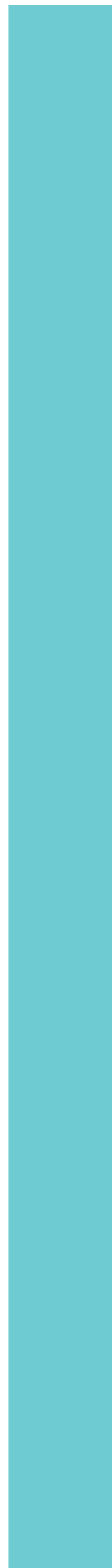

Fecha de recepción: diciembre 14 de 2012

Fecha de aceptación: agosto 2 de 2013

Received: December 14th, 2012

Accepted: January 29, 2013

Data de recepção: 14 de dezembro de 2012

Data de aceitação: 2 de agosto de 2013

Reçu le: 14 décembre, 2012

Accepté le: 2 août, 2013

RESUMEN

La creación de valor ha sido un reto permanente para la administración. A la eficiencia y la eficacia se agregaron la necesidad de crear valor al accionista. Las condiciones que han traído consigo la globalización, el predominio del capitalismo financiero y las TICs (Tecnologías de la Información y Comunicación), generan nuevos retos para los gobiernos, los empresarios y la academia; la administración ha buscado dar respuesta a dichos retos por medio de un nuevo modelo de gestión, el Gobierno Corporativo, GC. En el presente artículo se reflexiona en torno a las etapas

Master in Corporate Direction and Management, Universidad del Rosario, Colombia; Business Administrator, Universidad del Rosario, Colombia; Assistant Professor, Universidad del Rosario; Research Group Member in Corporate Durability, GIPE; rafael.pineros@ urosario.edu.co

Mestre em Direção e Gestãod e Empresas, Universidade de Rosario, Colômbia; Administrador de Empresas, Universidade de Rosario, Colômbia; Professor assistente, Universidade de Rosário; Grupo de pesquisa sobre perdurabilidade empresarial, GIPE; rafael.pineros@urosario.edu.co

M2 en Management et Gestion d'entreprise, Universidad del Rosario, en Colombie, Administrateur d'entreprise, Universidad del Rosario, Colombia, professeur adjoint, Université de Rosario groupe de recherche perdurable en entreprise, GIPE; rafael.pineros @ urosario.edu.co

* PhD. en Ciencias de la Dirección, Universidad del Rosario, Colombia; Magister en Administración, Universidad Externado, Colombia; Profesor auxiliar, Universidad del Rosario; Grupo de Investigación en Perdurabilidad Empresarial, GIPE; angela.noguera@ urosario.edu.co

$\mathrm{PhD}$ in Direction Science, Universidad del Rosario, Colombia; Master in Administration, Universidad Externado, Colombia; Assistant Professor, Universidad del Rosario; Research Group Member in Corporate Durability, GIPE; angela.noguera@urosario.edu.co

Ph.D em Ciências da Direção, Universidad del Rosario, Colômbia; Mestre em Administração, Universidad Externado, Colômbia; Professor assistente, Universidad del Rosário; Grupo de pesquisa sobre perdurabilidade empresarial, GIPE; angela.noguera@ urosario.edu.co

Doctorat en Sciences de Gestion, Universidad del Rosario, Colombia, Master en Management de I'Université Externado, en Colombie, professeure adjointe, Université de Rosario, groupe de recherche perdurable en entreprise, GIPE; angela.noguera @ urosario. edu.co 
de la administración y el surgimiento del GC, sus elementos constitutivos así como los retos que enfrenta. Se plantean conclusiones y se evidencia la necesidad de hacer seguimiento al desempeño de las organizaciones que adopten esquemas de GC.

PALABRAS CLAVE:

Gobierno, accionistas, stakeholders, valor

CLASIFICACIÓN JEL:

$\mathrm{M} 14$

ABSTRACT

The creation of value has been a permanent challenge for the administration. Added to efficiency and effectiveness, is the need to create value for the stockholder. The conditions brought by globalization, the predominance of financial capitalism and the ICT's (Information and Communication Technologies), generate new challenges for governments, business owners and the academy. Administration has looked for answers to such challenges through a new management model, the Corporate Government (CG). This article reflects around the administration stages and the emergence of the $C G$, its constituent elements as well as the faced challenges. Conclusions are proposed and a need for follow-up to the performance of organizations that adopt the CG schemes is evidenced.

Key words: Government, stockholders, stakeholders, value. JEL Classification: M14.

RESUMO

Criação de valor tem sido um desafio constante para a administração. À eficiência e eficácia foram adicionados a necessidade de criação de valor para o acionista. As condições que trouxe consigo a globalização, a predominância do capitalismo financeiro e as TICs (Tecnologias da Informação e Comunicação), geram novos desafios para os governos, empresários e a academia; a administração tem procurado responder a tais desafios através de um novo modelo de gestão, o governo corporativo, GC. Neste artigo, reflete-se sobre as etapas da administração e o surgimento do GC, seus elementos constituintes, bem como os desafios que enfrenta. Propõem-se conclusões e evidencia-se a necessidade de acompanhar o desempenho das organizações que adotam esquemas de GC.

Palavras chave: Governo, acionistas, agentes, valor. Classificação JEL: M14. 
La création de valeur a toujours été un défi pour l'administration. À l'efficience et à l'efficacité on doit ajouter la nécessité de créer une valeur actionnaire. Les conditions qui ont été apportées par la mondialisation, la domination du capital financier et des TIC (technologie d'information et de communication), génèrent de nouveaux défis pour les gouvernements, les entrepreneurs et les académies. L'administration a cherché à répondre à ces défis grâce à un nouveau modèle de gestion, le gouvernement coopératif, GC. Dans cet article on réfléchit aux étapes de l'administration et de l'émergence de la GC, ses éléments constitutifs et les défis auxquels elle est confrontée. On propose des conclusions et on évidence la nécessité de surveiller la performance des organisations qui adoptent des régimes GC.

Mots-clés: Gouvernement, actionnaires, partenaires, valeur. Classification JEL: M14.

\section{INTRODUCCIÓN}

El reciente caso de Interbolsa en Colombia tiene relación directa con el denominado Gobierno Corporativo, GC, el cual ha sido abordado por diversas ciencias sociales. El Derecho ha analizado el rol de los contratos y la teoría de agencia, la economía se ha enfocado en los temas relacionados con los costos y la sociología ha trabajado lo relativo a la cultura organizacional (Torres, Gorbaneff \& Contreras, 2008). En este mismo sentido la psicología ha estudiado lo relacionado con el comportamiento organizacional, y desde el Derecho, la administración y la filosofía se han estudiado temas como la ética corporativa y la responsabilidad social empresarial.

Al analizar la evolución de la administración se puede observar cómo el GC además de constituirse en una respuesta dada por el Estado, la academia y los mercados a los retos que han traído consigo los problemas derivados de las fallas inherentes al esquema de agencia y la economía de los costos de transacción, también puede considerarse como el resultado de un nuevo modelo de gestión que busca la creación de valor. El presente documento pretende describir, tomando como base la evolución de la administración y una serie de reflexiones planteadas por los autores, las diversas etapas que ha tenido la administración en su búsqueda por lograr la creación de valor, focalizándose en el GC como el actual modelo de gestión.

\section{ADMINISTRACIÓN Y CREACIÓN DE VALOR}

La Administración es un concepto que ha venido siendo construido a lo largo de los últimos cien años, razón por la cual el enfoque y contenido 
${ }^{6}$ En las organizaciones como máquinas se destaca el control y la optimización; como organismos el cambio, el desarrollo y la adaptación; como cerebros el aprendizaje organizacional y la Gestión del Conocimiento; como cultura los valores y creencias; y como sistemas de gobierno los conflictos de interés y el poder. que hoy tiene este término es marcadamente distinto al que poseyó en sus inicios. La administración eficaz de las empresas siempre ha implicado para la academia y los directivos un permanente interés por conocer con el mayor nivel de detalle posible cómo funcionan dichas organizaciones. En razón de la complejidad de las mismas, estos actores han utilizado una serie de metáforas que les han permitido demarcar de modo general el funcionamiento organizacional y diseñar perspectivas integrales al respecto. Las metáforas utilizadas por la dirección han sido: las organizaciones como máquinas, como organismos, como cerebros, como culturas y como sistemas de gobierno (Morgan, 1998).

En las organizaciones como máquinas se destaca el control y la optimización; como organismos el cambio, el desarrollo y la adaptación; como cerebros el aprendizaje organizacional y la Gestión del Conocimiento; como cultura los valores y creencias; y como sistemas de gobierno los conflictos de interés y el poder.

Según Magretta (2003) la misión de la administración es la creación de valor. Así las cosas, según esta autora todos los esfuerzos de la academia y los administradores han estado enfocados a dar respuesta a la pregunta: ¿Cómo crear valor? Para esto, la dirección y la academia han coincidido en diseñar una serie de modelos sustentados en diversos paradigmas, para los cuales se han empleado las metáforas antes referidas.

El oficio de administrar es tan antiguo como la especie humana; sin embargo, su desarrollo científico y profesionalización solo surgen hacia finales del siglo XIX, con los conceptos desarrollados por Frederick Taylor y Henry Fayol, los cuales estructuraron lo que hoy se denomina la administración científica. A partir de este momento Magretta (2003) describe una serie de etapas en las que la administración ha buscado dar respuesta al reto de crear valor.

La primera etapa que propone la autora la denomina Mentalidad del fabricante, la cual 
tuvo como fundamento central la idea de que la eficiencia era la respuesta única y suficiente para lograr la creación de valor requerida para la administración exitosa de las empresas. A partir de esta idea la optimización en el uso de los recursos fue la preocupación central, es decir, lograr el máximo beneficio posible por cada unidad de recurso utilizado. Es en esta etapa cuando la Administración Científica tiene su desarrollo; esta, apoyada en las ciencias exactas, propone una nueva concepción del trabajo y la producción.

La mentalidad del fabricante implicaba dirigir la mirada exclusivamente hacia el interior de la empresa; esto fue adecuado durante el tiempo en que los mercados se comportaron como oligopolios, es decir, que la demanda superaba a la oferta. Sin embargo, en la medida en que los mercados fueron cambiando, apareciendo nuevos competidores y nuevos clientes, la eficiencia como respuesta única para la generación de valor se tornó insuficiente.

Dadas las dificultades que empezaron a enfrentar las empresas que solo contaban con la eficiencia, surgió la Mentalidad del marketing. Para esta etapa la creación de valor se lograba garantizando no solo la optimización de los procesos y los recursos, sino que se hizo indispensable la eficacia, la cual exige mirar hacia fuera de la empresa; es un percibir y responder. En esta etapa aparece el interés por identificar las necesidades de los clientes y lograr que los productos y servicios de las empresas satisfagan apropiadamente dichas necesidades. A partir de esta etapa la estrategia empresarial adquiere una nueva dinámica dado que siendo la optimización fácil de imitar, las empresas deben empezar a redefinir sus planes, revisar y rediseñar su core bussiness, así como apoyarse decididamente en la innovación, el aprendizaje organizacional y la gestión del conocimiento, dado que en los nuevos mercados la competitividad se fundamenta no solo en la tecnología sino también en el capital intelectual de las compañías.

Esta economía del conocimiento trajo consigo para las empresas el reto en torno al desarrollo de estrategias centradas en garantizar la innovación, para lo cual las organizaciones empezaron a hacer grandes inversiones en tecnología, es decir, en la faceta hard de la gestión del conocimiento, y posteriormente en el fortalecimiento del capital humano o el elemento soft de la gestión del conocimiento (Chuang, 2004).

Posterior a la etapa de la Mentalidad del marketing, Magretta (2003) señala la Creación de Valor al Accionista como el nuevo mantra, haciendo referencia a la respuesta que debió dar la administración a la crisis desatada por las grandes quiebras corporativas relacionadas con Wall Street, las que se caracterizaron por ejecutivos que obtenían remuneraciones exorbitantes a pesar de que las compañías a su cargo generaban pérdidas para sus accionistas, y porque las empresas valían más cuando eran vendidas por partes que cuando se consideraban como un todo. Este momento es el antecedente inmediato del reconocimiento del Gobierno Corporativo como una nueva etapa en la administración, dado que es en este en donde surge la pregunta de a quién se debe el director de la organización, pregunta cuyo fundamento surge de los problemas de agencia.

A juicio de Barbosa, Contreras y Juárez (2010), posteriormente la administración ha venido transitando hacia una nueva etapa, a la que denominan Dirección Ética y Socialmente Responsable, en la cual la creación de valor exige además de eficiencia, eficacia e innovación, la satisfacción de todos los grupos que se ven afectados directa 0 indirectamente con el accionar de la organización (stakeholders). 


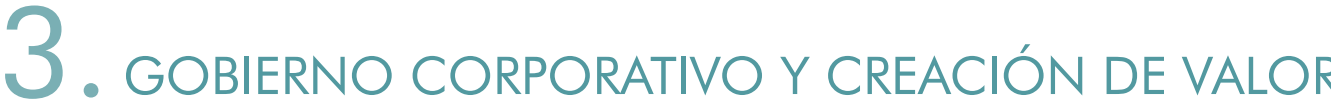

Tal como lo señala Hart (1995), el surgimiento del Gobierno Corporativo solo es posible si se dan dos condiciones. En primer lugar, que exista un problema de agencia que implica la generación de conflictos de intereses entre algunos de los siguientes agentes: propietarios, ejecutivos, miembros del consejo de administración, trabajadores o clientes. En segundo lugar, que los problemas asociados a los costos de transacción no puedan ser evitados o resueltos adecuadamente a través de los contratos.

Un problema de agencia surge cuando los propietarios (principal) deben confiar la administración de sus bienes a un gestor, mandante o gerente (agente), con el cual tendrá claras asimetrías de información, así como intereses distintos. El reto central es constituir mecanismos, generalmente contractuales, que prevean los incentivos y controles necesarios para que el agente busque proteger los intereses del principal, de tal modo que este no termine apoderándose de los bienes de los propietarios e inversionistas (Fernández, Gómez \& FernándezMéndez, 1999).

Como se puede observar al analizar la etapa denominada por Magretta (2003) Creación de Valor al Accionista, la autora está haciendo referencia a problemas de agencia surgidos de las acciones de una serie de administradores que al buscar satisfacer sus propios intereses terminan afectando los intereses de los propietarios. La creación de valor implica entonces, para los ejecutivos, buscar la eficiencia, la eficacia, la innovación y la satisfacción de los intereses de los propietarios. La cuestión entonces es estructurar un modelo que direccione al agente a proteger los intereses del principal, para lo cual el Gobierno Corporativo aporta los elementos centrales (Tovar, 2008).

Hoy día, dadas las lecciones aprendidas a través de las quiebras de empresas como ENRON y más recientemente Interbolsa, en Colombia, el Gobierno Corporativo comprende una serie de acciones que van más allá de lo financiero, llegando a incluir aspectos relacionados con el diseño de las organizaciones, las decisiones estratégicas, los sistemas de vigilancia y los esquemas de autorregulación, entre otras. Por lo anterior, cuando se menciona el tema de Gobierno Corporativo, ya no solo se incluyen acciones de cara a proteger al accionista y al inversor. Es así como en el Reino Unido, en 1992, el Comité de Cadbury a través del reporte acerca de los aspectos financieros del Gobierno Corporativo define el concepto como: "El sistema por el cual las compañías son dirigidas y controladas. Los consejos de administración son responsables por el gobierno de sus compañías" (Cadbury, 1992).

Surge la inquietud en torno a qué otros beneficios trae el GC además de proteger al accionista y al inversionista, así como la de preguntarse cuáles problemas adicionales resuelve. De acuerdo con el IFC (International Finance Corporation, 2005), unidad del Banco Mundial, el buen gobierno corporativo: permite disminuir el costo de la deuda, mejora la rentabilidad, ayuda a mantener a la compañía lejos de la corrupción y los malos manejos.

Es así como la financiación demandada por el crecimiento de importantes empresas, debe buscarse en medios distintos al bancario ya que en algunos casos el costo a corto plazo generado por las entidades crediticias no puede ser soportado por las empresas, aumentándose así el riesgo.

Un requisito para poder acceder a financiación en el mercado público de valores es la implementación de buenas prácticas de gobierno corporativo, que generen confianza en los inversores potenciales. Evidencia de lo expresado se encuentra en el estudio realizado por Villegas y Arbeláez (2004): 
"Los fondos de pensiones encuentran como factor determinante de la baja demanda por activos financieros del sector real en particular de acciones, la carencia de gobierno corporativo. Manifiestan su desconfianza hacia las nuevas empresas en el mercado de valores, debido a la deficiente calidad en la revelación y estandarización de la información. Por otro lado, mencionan los altos niveles de incertidumbre ocasionados por las malas experiencias en el pasado caracterizadas por la falta de control, supervisión y problemas en la difusión de la información. Factores que sólo a través de la implementación de un buen código de gobierno corporativo pueden solucionarse. Efectivamente, este aspecto está poco desarrollado en el país" (Villegas y Arbeláez, 2004, p. 61).

Por ello para las empresas que deseen crecer usando opciones alternativas de financiamiento, que permitan disminuir el costo de la deuda, es vital implementar prácticas de gobierno corporativo.

Una mayor rentabilidad es otro aspecto que se relaciona con el buen gobierno corporativo. En un estudio sobre los efectos financieros de la implementación de códigos de gobierno corporativo por parte de empresas colombianas, realizado por Franco \& Montalván (2010), se evidenció que el retorno sobre los activos luego de implementado el código mejora en más de $1 \%$ y que el efecto es amplificado dependiendo de la calidad del código, evidenciándose un incremento de $5 \%$ en los niveles de apalancamiento. Además se muestra que hay una relación positiva entre el aumento de las inversiones y el crecimiento en las ventas que se encuentra positivamente mediada por la presencia de códigos de buen gobierno. Se concluye del estudio, que las medidas del buen gobierno son apoyadas por los mercados financieros incrementando el suministro de capital a las empresas.

Al hacer a las compañías más responsables, transparentes con la información, gracias a diseño de estructuras y mecanismos de dirección y control, el gobierno corporativo disminuye la

\section{"... el Gobierno Corporativo}

comprende una serie de

acciones que van más allá

de lo financiero, llegando a

incluir aspectos relacionados

con el diseño de las

organizaciones, las decisiones

estratégicas, los sistemas de

vigilancia y los esquemas de

autorregulación, entre otras.

Por lo anterior, cuando se

menciona el tema de Gobierno

Corporativo, ya no solo se

incluyen acciones de cara

a proteger al accionista $y$

al inversor. 


\section{${ }^{66}$ La regla de la creación} de valor llama la atención frente a la necesidad de que tanto el consejo de administración como los ejecutivos tengan claro lo que significa maximizar el valor al accionista, teniendo en cuenta las características propias de cada uno de ellos. De hecho, la regla expresa claramente que los agentes deben crear valor para todos los accionistas. En este punto es fundamental la coherencia entre la creación de valor a largo plazo y el sistema de incentivos que se establezca para los agentes. probabilidad de que se presenten actuaciones corruptas o de inadecuada dirección.

Con el ánimo de entender por qué las empresas con Buen Gobierno Corporativo tienen una mejor valoración en bolsa que otras, Choi y Pae (2011) encontraron que el costo de capital de las empresas transadas en la Bolsa de Valores de Corea disminuye con el fortalecimiento de las prácticas de Gobierno Corporativo o de la ética empresarial, logrando aumentar así el valor de mercado de la compañía.

En realidad los beneficios del buen gobierno corporativo son múltiples; además de los descritos anteriormente, a continuación se enumeran algunos:

- La adopción de buenas prácticas de gobierno corporativo agrega valor generando confianza en los grupos de interés.

- La probabilidad de éxito de una empresa para obtener financiamiento a través del mercado de valores es mayor cuando tiene altos estándares de gobierno corporativo.

- El buen gobierno corporativo fideliza y atrae nuevos inversionistas.

- El buen gobierno corporativo aumenta la sostenibilidad de empresas familiares.

- El buen gobierno corporativo apoya la perdurabilidad empresarial.

- El buen gobierno corporativo ayuda a minimizar y solucionar los costos y problemas de agencia.

- Conflictos entre accionistas y administradores.

- Conflictos entre accionistas mayoritarios y minoritarios.

- Conflictos entre accionistas y otros grupos de interés.

- El buen gobierno corporativo disminuye el riesgo ético.

- El buen gobierno corporativo aumenta la percepción de confianza.

Tovar (2008) señala los factores clave del Gobierno Corporativo, destacando dentro de ellos, como es natural, una clara preocupación por los temas relativos al control de los agentes; 
sin embargo, como se pasa a precisar, también se consideran aspectos relacionados con la administración de las organizaciones y muy particularmente con la creación de valor. Son factores clave generales: la identificación de la sociedad, la regla de la creación de valor y el sistema de solución de conflictos de interés.

En cuanto a la identificación de la sociedad el autor resalta cómo el modelo de empresa determina el modelo de Gobierno Corporativo por aplicar. Se puede afirmar que el Gobierno Corporativo en su diseño e implementación debe respetar los aspectos organizacionales propios de la estrategia y el diseño organizacional.

La regla de la creación de valor llama la atención frente a la necesidad de que tanto el consejo de administración como los ejecutivos tengan claro lo que significa maximizar el valor al accionista, teniendo en cuenta las características propias de cada uno de ellos. De hecho, la regla expresa claramente que los agentes deben crear valor para todos los accionistas. En este punto es fundamental la coherencia entre la creación de valor a largo plazo y el sistema de incentivos que se establezca para los agentes.

El sistema de solución de conflictos de interés guarda relación con los sistemas de repartos de beneficios y los conflictos que pueden surgir entre accionistas y accionistas, agentes y accionistas, y agentes entre sí. Este sistema debe soportar los procesos de toma de decisiones en el interior de la organización y debe contar con los mecanismos que garanticen la trasparencia y publicidad necesarias para el buen gobierno de la empresa.

Como factores clave especiales se señalan: política de tratamiento de la información, política de integración del consejo de administración, sus comités y la gerencia, política de remuneración para ejecutivos y miembros del consejo y política de dividendos.

Dentro de la política de tratamiento de la información es necesario precisar cómo se regula el acceso a la información y la comunicación de la misma a los diferentes stakeholders, de cara al hecho de que la información es elemento central y determinante para la toma de decisiones de accionistas, consejeros, ejecutivos, trabajadores, clientes e inversores.

En cuanto a la política de integración del consejo de administración, sus comités y la gerencia, es preciso tener claridad en cuanto al sistema de selección y a las funciones de cada actor. Se debe garantizar la independencia e idoneidad profesional y personal, así como la experiencia necesaria, con la finalidad de contar con un consejo y ejecutivos profesionales en su rol.

La retribución de consejeros y ejecutivos debe permitir que el agente se alinee con los intereses del principal, y a la vez impedir que el primero se apodere del patrimonio de los accionistas e inversionistas. Esta política debe ser consistente con las definiciones estratégicas que guíen el accionar de la empresa y con la presentación y difusión que de la misma se haga al mercado.

En cuanto a la política de dividendos se resalta la necesidad de contar realmente con dicha política, lo cual implica contar con una política específica, clara y detallada, que contemple los aspectos relativos a la repartición de los mismos. Debe así mismo guardar plena coherencia con los aspectos estratégicos de la compañía y con los demás factores clave del Gobierno.

Estos factores clave generales y especiales del gobierno corporativo, que giran a favor de aliviar las problemáticas alrededor de la alineación de intereses entre el agente y el (los) principales y de la teoría económica de la firma que le otorga personería jurídica y responsabilidad limitada a los inversores; deben ampliarse de modo que incluyan nuevas perspectivas como son: la de creación de valor para los grupos de interés, el desarrollo sostenible y la globalización de los mercados de capital. 


\section{RETOS Y TENSIONES ESTRUCTURALES DEL 4. GOBIERNO CORPORATIVO}

A continuación se presentan los retos que enfrentan los directivos y miembros de las juntas directivas en torno al diseño e implementación de las acciones propias del Gobierno Corporativo, y se reflexiona en torno a lo que Barbosa, Medina y Vargas (2013) plantean como los retos estructurales que surgen entre el GC, la responsabilidad social y la globalización.

\subsection{CREACIÓN DE VALOR: ¿PARA QUIÉN?}

Ya que el gobierno corporativo se preocupa por establecer la forma en que se dirigen y controlan las compañías, un aspecto crítico por determinar es el propósito de este tipo de organizaciones. Como se ha anotado antes, la administración en sus etapas iniciales y muy recientes ha tenido claro que el criterio de desempeño de la organización de tipo empresarial es maximizar el valor para el accionista e inversionista, y en consecuencia ha establecido sistemas de medición y control como la estructura de información financiera y los métodos de valuación empresarial.

Sin embargo, desde que en los años 70 el Premio Nobel de Economía Milton Friedman afirmó que "la única responsabilidad social de los negocios es maximizar las utilidades" (Friedman, 1970), se viene presentado un debate sobre los propósitos corporativos. Es así como la teoría de los grupos de interés (stakeholder theory), desarrollada por Freeman (1984) considera esta postura como limitada al dar prioridad a los intereses de los accionistas por encima de los de otros grupos de interés.

Fruto de los escándalos corporativos que ocurrieron en Estados Unidos durante los años 2001 y 2002, Sundaram \& Inkpen (2004) hicieron una revisión del debate acerca de los propósitos corporativos y concluyeron que la maximización de valor para el accionista es la mejor opción, ya que las críticas relacionadas con los incentivos de transferencias y las implicaciones de las fallas contractuales no son exclusivas de los accionistas, sino que pueden suceder con cualquier grupo de interés. Frente a esta postura Freeman, Wicks y Parmar (2004) concluyeron que la meta de crear valor para los grupos de interés mejora la creación de valor para los accionistas, a su vez crea incentivos apropiados para que los directivos asuman riesgos empresariales, y clarifica la elección de los medios para el logro de los objetivos. En suma, es claro que hoy día el objetivo es la creación de valor compartido para la corporación, sus grupos de interés y en general, la sociedad.

Es así como el concepto de RSE, que implica la acción voluntaria de la empresa para comprometerse con el desarrollo de sus grupos de interés y no solo de sus accionistas, se vincula con el concepto de Gobierno Corporativo. En una investigación que incluyó información de más de 3.000 compañías entre 1993 y 2004, Jo y Harjoto (2011) estudiaron la relación entre Gobierno Corporativo, responsabilidad social corporativa y desempeño financiero. Los resultados muestran que la implementación de prácticas de Gobierno Corporativo tiene un efecto positivo en la ejecución de prácticas de responsabilidad social; a su vez, se evidencia que las corporaciones que llevan a cabo prácticas de responsabilidad social a través de la gestión de las relaciones con los grupos de interés, se desempeñan mejor en términos de rentabilidad y maximización de bienestar para los accionistas.

Es así como el diseño de mecanismos para lograr que la corporación sea capaz de crear valor para todos los grupos de interés es un nuevo reto para el Gobierno Corporativo. Las estructuras de poder y de decisión que se dan en el interior de los órganos directivos deben incorporar las preocupaciones de la responsabilidad social corporativa como un imperativo estratégico. 


\subsection{GOBIERNO CORPORATIVO Y DESARROLLO SOSTENIBLE}

La Comisión Mundial para el Ambiente y el Desarrollo de las Naciones Unidas en 1987 acuñó la definición clásica de desarrollo sostenible: "un desarrollo que satisface las necesidades del presente sin comprometer la habilidad de generaciones futuras de satisfacer sus propias necesidades". Se trata de un principio que une tres pilares: desarrollo económico, equidad social y protección ambiental.

Eventos como el de Río +20 son prueba de que el nivel de conciencia sobre las consecuencias de las actuales formas de producción y explotación ha aumentado; por ello es evidente que para las compañías es imprescindible la adopción de estrategias que se encuentren en armonía con las proposiciones del desarrollo sostenible.

De acuerdo con el análisis hecho por Kruze \& Lundbergh (2010), el Gobierno Corporativo y el desarrollo sostenible son interdependientes, más de 411 inversionistas han firmado los Principios para la inversión responsable de las Naciones Unidas (UNPRI) y se encuentra evidencia de que varias estructuras de Gobierno Corporativo de grandes compañías incluyen comités de sostenibilidad.

De la misma manera se encuentra que el valor de mercado de las firmas que pertenecen al S\&P 500 entre 1999 y 2002 en Estados Unidos y que llevan a cabo prácticas de desarrollo sostenible, esto es, que tienen en cuenta aspectos económicos, ambientales y sociales en el desarrollo de sus estrategias, aumenta según estudios publicados por Lo y Sheu (2007). Por esta razón se espera que las prácticas de Gobierno Corporativo y de responsabilidad social involucren cada vez más los imperativos del desarrollo sostenible.

A continuación se resumen los hallazgos de una investigación realizada por Ricart, Rodríguez \& Sánchez (2005), que tuvo como objetivo describir cómo los sistemas de GC están evolucionando en la integración de las prácticas de desarrollo sostenible, para lo cual analizaron los sistemas

\section{6 ... la meta de crear valor}

para los grupos de interés mejora la creación de valor para los accionistas, a su ver. crea incentivos apropiados para que los directivos asuman riesgos empresariales, y clarifica la elección de los medios para el logro de los objetivos. En suma, es claro que boy día el objetivo es la creación de valor compartido para la corporación, sus grupos de interés y en general, la sociedad. 
66 ... existen varios modelos de gobierno corporativo en el mundo que difieren del grado de capitalismo existente. El modelo liberal anglosajón tiende a dar prioridad a los intereses de los accionistas, mientras que el modelo coordinado de Europa continental reconoce además los intereses de los trabajadores, directivos, proveedores, clientes y la comunidad. Por ello los sistemas legales relacionados con las prácticas de gobierno corporativo difieren de un continente a otro. de gobierno de 18 compañías líderes en el Dow Jones Sustainability World Index (DJSI World). Con base en lo anterior desarrollaron un modelo de Gobierno Corporativo sostenible basado en las teorías de desarrollo sostenible, Gobierno Corporativo y los resultados obtenidos a partir del análisis hecho a los datos de las compañías.

El modelo de Gobierno Corporativo sostenible desarrollado por los autores responde a los siguientes aspectos: quiénes deberían ser los miembros del consejo de administración, cuáles serían sus roles más importantes, cómo debería funcionar el consejo para lograr la efectiva ejecución de los roles y por qué debería hacerse.

El por qué debería hacerse, obedece al replanteamiento de que la maximización de valor para el accionista no debería ser la única meta de las compañías; la meta debería ser contribuir a la sostenibilidad de la vida en el planeta, es decir, lograr el desarrollo sostenible.

Los miembros del consejo de administración deben tener un fino entendimiento de los requisitos y retos del desarrollo sostenible y la responsabilidad social. Para lograrlo incluyen en el consejo expertos en estos temas y proveen entrenamiento a sus directivos.

Para que el funcionamiento del consejo sea efectivo en el logro de las metas del desarrollo sostenible y la responsabilidad social, la estructura debe adaptarse para que las metas puedan ser discutidas y trabajadas de la misma forma que otras que han sido tradicionales.

Los roles identificados para el consejo son: involucramiento con grupos de interés, promoción de valores clave, inclusión del desarrollo sostenible y la responsabilidad social en la estrategia corporativa, y evaluación del desempeño en estas áreas.

Sin embargo, como concluyen los autores, para lograr avanzar hacia la sostenibilidad es necesario aproximarse desde la innovación, que es una forma de lograr ventajas competitivas más 
sostenibles. Por ello se considera que uno de los factores de éxito más importantes para que el GC logre asumir los retos de la sostenibilidad es la innovación.

\subsection{LA GLOBALIZACIÓN DE LOS MERCADOS DE CAPITAL}

Como analizan Ping y Cheng (2011), existen varios modelos de gobierno corporativo en el mundo que difieren del grado de capitalismo existente. El modelo liberal anglosajón tiende a dar prioridad a los intereses de los accionistas, mientras que el modelo coordinado de Europa continental reconoce además los intereses de los trabajadores, directivos, proveedores, clientes y la comunidad. Por ello los sistemas legales relacionados con las prácticas de gobierno corporativo difieren de un continente a otro.

Así mismo, de acuerdo con la revisión hecha por estos autores actualmente se presenta una tendencia al desarrollo de mercados de capital globalizados y, en consecuencia, a la convergencia alrededor de las problemáticas que se dan entre los inversores y los responsables de las corporaciones a nivel mundial; por ello los Estados, reguladores, bolsas de valores e inversores institucionales se preparan para la homogenización de los estándares de gobierno corporativo.

Por otro lado, según Yuksel (2008) en la economía global, compañías y consumidores exigen cada vez más dos cosas: confianza internacional estandarizada y aplicación de procedimientos adecuados. Del análisis del caso de Turkía, país que ha venido ajustando su regulación a los estándares internacionales, concluyen que la inversión extranjera aumenta en aquellas firmas que son más compatibles con los estándares internacionales.
Con relación a lo anterior se afirma que existen cuatro tensiones estructurales que explican las dificultades y retos que vienen enfrentando empresas, Estado y sociedad al buscar la aplicación del GC y la RS en un mundo globalizado (Barbosa, Medina \& Vargas, 2013):

1) El deber del Estado de garantizar derechos a los ciudadanos dentro de su territorio, frente a la dependencia existente por los grandes grupos económicos y agentes de capital, de origen privado o público, nacional o trasnacional; 2) El deber del sistema económico de ofrecer igualdad de oportunidades basadas en el trabajo y el ánimo de lucro, frente a un modelo de creación de la riqueza basado en el beneficio segmentado, la especulación y el riesgo; 3) El derecho de propiedad equitativo para todo ciudadano en modelos democráticos, frente a la concentración exagerada de recursos en poder de una minoría; y 4) El deber de la empresa ante el modelo económico y la obligación ética de asumir voluntariamente políticas de responsabilidad social empresarial (Barbosa, Medina \& Vargas, 2013, p. 41).

Se afirma entonces que el papel del GC ha venido evolucionando al redefinir su objetivo, dado que hoy se busca con el mismo, además de su tradicional función de proteger al accionista e inversionista, impactar positivamente en la satisfacción de las necesidades y la atención de las preocupaciones de todos los agentes que operan en cualquier sector de la economía. Será necesario entonces seguir observando hacia dónde se siguen encaminando los esfuerzos en materia de GC y la dirección de las organizaciones, dado que el riesgo que se corre es que los Estados terminen transfiriendo a las empresas una serie de acciones y responsabilidades que por naturaleza deben permanecer en cabeza de este. 
La investigación realizada confirma que el Gobierno Corporativo se constituye como un prometedor modelo de gestión para la generación de valor compartido. Los múltiples beneficios que trae no solo para la compañía sino para todos sus grupos de interés, confirman que es una estrategia clave en la construcción de empresas perdurables.

Se evidencia también que las estrategias y mecanismos que actualmente hacen parte del Gobierno Corporativo son cada vez más eficaces en la protección al accionista (principal) frente a los comportamientos indebidos que pueda tener el administrador de la compañía (agente); y que han logrado una mayor profesionalización de la administración y un aumento en la transparencia empresarial.

Los estudios revisados demuestran además una relación causal positiva entre la implementación de prácticas de Gobierno Corporativo y el desarrollo de iniciativas de responsabilidad social y de ética empresarial; actividades que, como también se evidenció, logran disminuir el costo de capital, aumentando a su vez el valor de mercado de las compañías que lo practican. Por ejemplo, para el caso colombiano, se presenta evidencia de que la implementación de códigos de buen gobierno de alta calidad logra aumentar la tasa de retorno sobre activos.

Adicionalmente se identificaron tres grandes retos en el desarrollo del Gobierno Corporativo. Por un lado, la reformulación del objetivo corporativo exige una evolución de las estructuras y mecanismos que apunten a la creación de valor no solo para el accionista, sino además para todos los grupos de interés; cumpliendo de esta forma con la requisitos y exigencias de la responsabilidad social corporativa.
Por otro lado, las preocupaciones por la construcción de un mejor futuro común, en donde se tengan en cuenta los intereses de las futuras generaciones, demanda orientar el Gobierno Corporativo a las exigencias del desarrollo sostenible. Las recomendaciones para incorporar el desarrollo sostenible en el Gobierno Corporativo de las corporaciones incluyen el alineamiento con la estrategia a través de la formulación de objetivos de sostenibilidad, la inclusión de un director de sostenibilidad en la junta directiva, el involucramiento con grupos de interés, la inclusión de indicadores de sostenibilidad en los sistemas de gestión de riesgos, la invitación de expertos al consejo de administración, el entrenamiento de directivos, el diseño de estructuras de incentivos, y la ejecución de estrategias de divulgación.

La diversidad de modelos de gobierno corporativo en el mundo frente a la globalización de la economía impone el tercer reto identificado. La preocupación de inversionistas y consumidores entre otros grupos de interés por la recuperación de la confianza en las corporaciones y el aseguramiento de una adecuada gestión de las mismas; implica el desarrollo de estándares de Gobierno Corporativo internacional que puedan ser adaptados a condiciones locales.

Finalmente, se confirma la pertinencia de la aplicación del GC con base en la evidencia que permite identificar la generación de valor compartido, lo cual impacta positivamente en la superación de los retos ya descritos. Se hace necesario, sin embargo, adelantar estudios que monitoreen el comportamiento de los indicadores que permitan vigilar si los problemas que se busca resolver a través del GC efectivamente tienden a resolverse. 


\section{BIBLIOGRAFÍA}

Barbosa, D.; Contreras, F.V.; Juárez. F. (2010). Recursos emocionales para la dirección de organizaciones. Manuscrito presentado para su publicación.

Barbosa, D.; Medina, C.; Vargas (2013). Globalización, capitalismo financiero y responsabilidad social empresarial: Tensiones estructurales. Tesis de maestría no publicada. Universidad del Rosario, Bogotá, Colombia.

Cadbury (1992). Report of the committee on the Financial Aspects of Corporate Governance, p. 14; University of Cambridge, UK.

Choi, T. \& Pae, J. (2011), Corporate Governance, Commitment to Business Ethics, and Firm Valuation: Evidence from the Korean Stock Market. Journal of Business Ethics, 100, pp. 323-348.

Chuang, S. (2004). A resource-based perspective on knowledge management capability and competitive advantage: An empirical investigation. Expert Systems with Applications, 27, pp. 459-465.

Fernández, A.; Gómez, S.; Fernández-Méndez, C. (1999). El papel supervisor del consejo de administración sobre la actuación gerencial. Evidencia para el caso español. Investigaciones Económicas, 22 (3), pp. 501-516.

Franco, J.; Montalván, S. (2010). Governance Codes: Facts or Fictions? A study of governance codes in Colombia. Estudios Gerenciales, Vol. 26, No. 117, pp. 85-102.

Freeman, E. (1984). Strategic Management: A stakeholder approach. Boston: Pitman.

Freeman, E.; Wicks, A.; Parmar, B. Stakeholder Theory and "The Corporate Objective Revisited"; Journal Organization Science, Vol. 15, No3, pp. 364-369.

\section{6 ... el Gobierno Corporativo} se constituye como un prometedor modelo de gestión para la generación de valor compartido. Los múltiples beneficios que trae no solo para la compañia sino para todos sus grupos de interés, confirman que es una estrategia clave en la construcción de empresas perdurables. 
Friedman, M. (1970). "The social responsability of Business is to Increase its Profits". New York Times Magazine (13 September).

Harjoto, M. (2011). The causal effect of Corporate Governance on Corporate Social Responsibility. Journal of Business Ethics, 106, pp. 53-72.

Hart, O. (1995). Corporate Governance: some theory and implications. The Economic Journal, pp. 678-689.

International Finance Corporation (2005). The Irresistible Case for Corporate Governance. Disponible en: www.ifc.org

Kruse, C.; Lundbergh, S. (2010). The governance of Corporate Sustainability. Rotman Journal of Pension Management, Vol. 3, Issue 2.

Lo, S.; Sheu, H. (2007). Is Corporate Sustainability a Value-Increasing Strategy for Business? Journal Compilation. BlackWell Publishing Ltd., Oxford, USA, Vol. 15, No. 2.

Magretta, J. (2003). Qué es management: cómo funciona y por qué nos afecta. Colombia: Empresa Activa.

Morgan, G. (1998). Images of Organizations. San Francisco: Yale University Press.

Ping, Z.; Cheng, A. (2011). Corporate Governance: A summary review on Different
Theory Approaches. International Research Journal of Finance and Economics, Issue 68, pp. 7-13.

Ricart, J.; Rodríguez, M.; Sánchez, P. (2005). Sustainability in the boardroom: An empirical examination of Dow Jones Sustainability World Index. Corporate Governance, p. 24.

Sundaram, A.; Inkpen, (2004). The Corporate Objective Revisited. Organization Science, Vol. 15, No. 3, pp. 350-363.

Torres, S.; Gorbaneff, Y.; Contreras, N. (2005). Prioridades en la definición de límites entre aseguradores y prestadores de servicios de salud. Revista Gerencia y Políticas de Salud, pp. $141-158$.

Tovar, J. (2008). Las claves del gobierno corporativo. Revista de Economía y Derecho, pp. 41-60.

Villegas, F.; Arbeláez, M. (2004). Colombia: Diagnóstico de la estructura de Financiamiento del Sector Real e Identificación de Obstáculos que han impedido que estas empresas acudan al Mercado de Valores.

Yuksel, C. (2008). Recent developments of Corporate Governance in the Goblal Economy and the New Turkish Commercial Draft Law Reforms. Journal of International Commercial Law and Technology, Vol. 3, Issue 2. 